\title{
A Comparative Study on the Activity and Antigenicity of Truncated and Full-Length forms of Streptokinase
}

\author{
REZA ARABI ${ }^{1}$, FARZIN ROOHVAND $^{1,2 *}$, DARYOUSH NOROUZIAN ${ }^{3}$, SOROUSH SARDARI $^{4}$, \\ MOHAMMAD REZA AGHASADEGHI ${ }^{1}$, HOSEIN KHANAHMAD ${ }^{5}$, ARASH MEMARNEJADIAN ${ }^{1}$ \\ and FATEMEH MOTEVALLI ${ }^{1,2}$
}

${ }^{1}$ Hepatitis and AIDS Dept., ${ }^{2}$ NRGB,${ }^{3}$ Bacterial vaccine Dept., ${ }^{4}$ Biotechnology Dept.
${ }^{5}$ BCG vaccine Dept., Pasteur Institute of Iran

Received 15 February 2011, revised 30 April 2011, accepted 30 May 2011

\author{
Abstract
}

\begin{abstract}
Application of streptokinase (SK) as a common and cost-effective thrombolytic drug is limited by its antigenicity and undesired hemorrhagic effects. Prior structural/functional and epitope-mapping studies on SK suggested that removal of $59 \mathrm{~N}$-terminal residues led to its fibrin dependency and identified SK antigenic regions, respectively. Following in silico analyses two truncated SK proteins were designed and compared for their fibrin specificity and antigenicity with the full-length SK. Computer-based modeling was used to predict the effect of vector (pET41a)-born protein tags on the conformation of SK fragments. SK60-386, SK143-386 and full-length SK (1-414) were separately cloned, expressed in BL21 E. coli cells and confirmed by Western-blotting. Functional activity of the purified proteins was evaluated with chromogenic and clot lysis assays and their antigenicity was tested by ELISA assay using rabbit anti-streptokinase antibody. As expected, chromogenic bioassay showed a major activity decline for SK60-386 and SK143-386 (83 and 91 percent, respectively), compared to SK1-414. However, in clot lysis assay, which is a fibrin-dependent pharmacopoeia-approved test, SK60-386 and SK143-386 were respectively 35 and 31 percent more active though lysed the clots slower than full-length SK. Antigenic analysis also indicated significant decrease in ELISA signals obtained for truncated proteins compared to SK1-414 (45 and 28 percent less reactivity for SK143-386 and SK60-386, respectively, $p<0.0001$ ). The results of this study for the first time pointed to SK143-386 and SK60-386, as improved SK derivatives with increased fibrin-selectivity and decreased antigenicity as well as acceptable bioactivity profiles in a pharmacopoeia-based analysis, which deserve more detailed pharmacological studies.
\end{abstract}

Ke y words: antigenicity, fibrin specificity, protein modeling, truncated streptokinase

\section{Introduction}

Several thrombolytic drugs (plasminogen activators; PAs) with different pharmacokinetic and pharmacodynamic properties have been developed for treating diseases such as stroke, pulmonary embolism, deep vein thrombosis and acute myocardial infarction, among which streptokinase (SK) and tissue plasminogen(Plg) activator (tPA) are the most commonly used agents (Banerjee et al., 2004; Baruah et al., 2004). Although no predominant thrombolytic drug has been introduced to be used for every indication so far (Banerjee et al., 2004), however, several clinical trials comparing the efficacy of SK and tPA generally suggested that streptokinase is the drug of choice for thrombolytic therapy especially in resource limited countries (Banerjee et al., 2004; Baruah et al., 2004).

Streptokinase is produced by different strains of $\beta$-hemolytic streptococci and due to its non human origin, is immunogenic and can evoke the immune system; hence, frequent administrations of SK can result in production of neutralizing antibodies, which in turn reduces the efficacy of therapy, and eventually may lead to extensive allergic reactions (Banerjee et al., 2004; Baruah et al., 2004; Parhami-Seren et al., 1997; Parhami-Seren et al., 1995; Reed et al., 1993; Torrèns et al., 1999). Besides, SK function is not fibrin-specific; therefore its infusion to patients' blood may result in rapid activation of plasminogen and subsequent ectopic emergence of plasmin in circulatory system. This unspecific action of SK has undesirable consequences such as general depletion of plasminogen (Plg) concentration in circulatory system, rapid production of bradykinin resulting in hypotension (Reed et al., 1999) and elevating the risk of hemorrhage (Banerjee et al., 2004; Baruah et al., 2004; Mundada et al., 2003; Reed et al., 1999; Sazonova et al., 2004). Therefore, there is high interest in providing modified or truncated SK molecules with improved fibrin-dependency and less immunogenicity.

\footnotetext{
* Corresponding Author: F. Roohvand, Hepatitis and AIDS Dept. and NRGB, Pasteur Institute of Iran, Pasteur Ave., Tehran 1316943551, Iran; phone/fax: +98.21.66969291; e-mail: rfarzin@pasteur.ac.ir
} 
Streptokinase with a molecular mass of $47 \mathrm{kDa}$, is a 414 amino acid single strand peptide which lacks cystine, cystein, phosphorous, conjugated carbohydrates and lipids (Banerjee et al., 2004). SK forms an equimolar complex with plasminogen or plasmin and this complex converts plasminogen substrates to plasmin by hydrolyzing the amide bound between $\operatorname{Arg}_{561}$ and $\mathrm{Val}_{562}$. Eventually the resulted plasmin can degrade and solubilize the fibrin clots (Wang et al., 1998). Crystallography analysis of streptokinase structure (Wang et al., 1998) has shown that it is composed of 3 domains known as $\alpha, \beta$ and $\gamma$ (residues 12-150, 151-287 and 287-372 respectively) domains (Reed et al., 1999). All three domains of the protein are involved directly or indirectly in the SK interaction with Plg (Conjero-Lara et al., 1998). $\gamma$ domain which has a close contact with Plg active site (Wang et al., 1998) plays a central role in amidolytic activity of the activator complex (ConjeroLara et al., 1998) while $\beta$ domain is known as the region responsible for high affinity interaction between SK and $\mathrm{Plg}$. Although the exact role of $\alpha$ domain is not clearly defined, but the first 59 residues of this domain are important for streptokinase accurate conformation and full activity (Shi et al., 1994). In fact, deletion of these first 59 amino acids of the $\mathrm{N}$-terminal segment (SK $\Delta 59$ or SK60-414) resulted in major reduction of SK activity in the absence of fibrin, but interestingly in the presence of fibrin the activity was shown to be restored albeit in a longer period of time. In other words, SK $\Delta 59$ showed a fibrin-dependent full-activity characteristic when SK-Plg reaction were awaited for longer times (Mundada et al., 2003; Sazonova et al., 2004). Further studies indicated that presence of plasmin is an essential element for efficient activation of SK $\Delta 59$ (Mundada et al., 2003; Sazonova et al., 2004). In fact, addition of trace amounts of plasmin markedly increased plasminogen activity of SK $\Delta 59$ and declined the previously reported lag time of the reaction. Moreover, in contrast to the wild type SK which protected plasmin from inhibition by $a_{2}$-antiplasmin, SK $\Delta 59$ is more prone to such haemostatic regulations to prevent the risk of hemorrhage. Therefore, since in the real physiological condition, only plasminogen substrates on the surface of fibrin can be fully activated by SK60-414* plasmin complex (Mundada et al., 2003; Reed et al., 1999; Sazonova et al., 2004) and just fibrin-bound plasmin is protected from inhibitory action of $\alpha_{2}$-antiplasmin (Mundada et al., 2003), thus this truncated form of streptokinase was considered as a fibrin targeted plasminogen activator and a potentially improved SK for thrombolytic therapy (Mundada et al., 2003; Sazoana et al., 2009; Sazonova et al., 2004). However, to our knowledge there is no prior study addressing a standard pharmacopoeia comparative analysis based on clot lyses assay between full length SK and SK $\Delta 59$ in allowed reaction period of times to approve the efficiency of this truncated SK as a thrombolytic drug.
It is also well shown that during in vivo and in vitro $\mathrm{Plg}$ activation, SK is cleaved into three fragments spanning amino acid residues of 1-59, 60-386 and 387-414 (Reed et al., 1999). While the N-terminal fragment remains tightly attached to the main segment, the C-terminal part, i.e. amino acids $387-414$ is released during Plg activation, implying that this part may not be important for SK activity (Reed et al., 1999). Although the SK60-386 may be even a potentially more improved fibrin-dependent PA drug because of losing some immunogenic segments of SK located in its C-terminal (compared to SK60-414) but no prior study has addressed evaluation of its activity by fibrin based methods either.

It was previously demonstrated that 143-386 fragment of streptokinase has only 60 percent of maximal activity over even a longer period of time compared to both full-length streptokinase and SK60-414 (Rodriguez et al., 1995). This fragment lacks the so-called nonessential C terminal part similar to SK60-386 and moreover has an extra 83 residue deletion in the $\mathrm{N}$ terminal region. Although SK143-386 has a dramatic reduction of activity in the absence of fibrin, but fibrin degradation potential of this fragment (which lacks more immunodominant regions of SK) or changes of its activity in the presence of fibrin was not previously studied either.

Different regions of streptokinase have been identified as antigenic epitopes by using murine and human monoclonal antibodies as well as sera of patients treated with SK. Many of these regions, including residues 4 to 8 and 96-99 (Coffey et al., 2001), 120-140 (ParhamiSerena et al., 2003), 353-414 (Reed et al., 1993) and 373-414 (Torrèns et al., 1999), are located in regions which are marked out for SK truncations in our study (that is 1-59,1-142 and 387-414) with the expectation that removal of these parts may result in the reduction of antigenicity in truncated SK molecules.

In the present study, with the final aim of improving the potential therapeutic efficacy of streptokinase by development of more fibrin-specific SKs with less antigenicity, we sought to produce two recombinant truncated streptokinase derivatives spanning the residues of either $143-386$ or $60-386$ and analyzed their clot lyses activity by a standard pharmacopoeia fibrinbased method with that of full length SK. We also provide comparative data for improved and less antigenic profiles of these truncated molecules.

\section{Experimental}

\section{Materials and Methods}

Computer-based modeling. To evaluate the impact of the protein tags derived from pET4la (used for expression of SKs and its derivatives in this study) on the conformation of full length and truncated forms of 


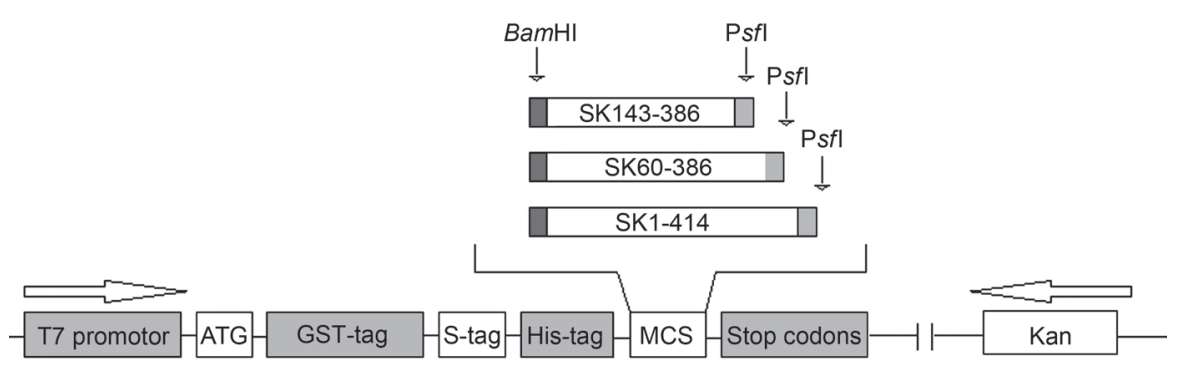

Fig. 1. Schematic illustration for insertion site of 3 different forms of $s k c$ in pET41a vector.

Truncated (143-386 and 60-386) and full-length(1-414) forms of skc were inserted into BamH1 and Pst1 sites of MCS of pET41a; ATG and Kan stands for start translation codon and kanamycin resistance respectively; MCS stands for for multiple cloning sites; GST-tag, S-tag and His-tag are the tags derived from the vector.

SKs, three-dimensional structure of the SK proteins fused to the vector derived-tags were modeled using MODELLER program (version 9v5, 2008) which is a non-graphical, command-line based software. To this end, 1 bmlC.pdb and $1 \mathrm{~m} 9 \mathrm{~A} . p d b$ atom files (as templates for streptokinase and GST proteins respectively) were downloaded from Protein Data Bank (http://www.rcsb. org) and multiple template script files of MODELLER program were used for modeling. Visualization of the models was performed by Deep View Spdb-viewer (version 4.0.1, 2009) and WebLab viewer Lite (version 4, 2000) softwares. The resulted models were evaluated based on three methods: 1. Energy level comparison: in which energy calculation of residues of model and template was performed by applying DOPE potential command line of MODELLER program and energy level of the residues were compared in a graphing program. 2. Root Mean Square Deviation (RMSD) calculation: in which the 3D structures of templates and models were superimposed in Deep View spdb-viewer program (version 4.0.1, 2009) and the quality of the fits were evaluated by calculating Root Mean Square Deviation (RMSD) between carbon $\alpha$ of the residues. 3. Ramachandran plot assessment: which was used to visualize dihedral angles $\psi$ against $\varphi$ of amino acid residues in protein structure by using RAMPAGE server (Lovell et al., 2002).

Bacterial strains and culture media. Plasmid propagation and preparation was performed in E. coli DH5a (Novagen, USA) and E. coli BL21 (DE3) (Novagen, USA) was used for protein expression of cloned genes by IPTG induction. Bacteria were cultivated in LB (Luria-Bertani) broth or TY2X for plasmid extraction and expression induction, respectively. All strains were stored in $20 \%(\mathrm{v} / \mathrm{v})$ glycerol and stab culture of LB Agar.

Gene amplification, cloning and expression. Streptokinase gene $(s k c)$, from a standard streptococcus strain (S. equisimilis, ATCC 9542) which is also known as skc2 (Estrada et al., 1992), was PCR amplified by Pfu DNA polymerase. Various BamHI-tailed forward and Pst I-tailed reverse primers (Table I) were used to amplify the nucleotide sequences corresponding to full length (SK1-414) and truncated SKs (SK60-386, SK143-386). In this regard Pf1 and Pf414 were used as primers for amplification of $s k c 1-414$ and pairs of Pf60, Pf386 and Pf143, Pf386 were applied for amplification of $s k c 60-386$ and $s k c 143-386$, respectively. Thermal program was set as $30 \mathrm{cycles}$ of $95^{\circ} \mathrm{C}$ for $60 \mathrm{sec}, 58^{\circ} \mathrm{C}$ for $45 \mathrm{sec}$ and $72^{\circ} \mathrm{C}$ for $90 \mathrm{sec}$, which was followed by a final extension at $72^{\circ} \mathrm{C}$ for $5 \mathrm{~min}$.

The amplicons, after digestion with BamHI/PstI restriction enzymes, were separately cloned into the same sites of pET41a vector (Novagen, USA) downstream and in frame of the vector-derived tags (GST, $\mathrm{S}$ and His-tag), under the control of a T7 promoter (Fig. 1) to construct three recombinant plasmids of pETSK1, pETSK60 and pETSK143. The constructs which respectively encoded SK1-414, SK60-386 and SK143-386 were confirmed by sequencing (SeqLab, Germany) and subsequently were transformed into the E. coli BL21 cells. Single colony transformants were

Table I

Nucleotide sequence of primers designed for amplification of truncated and full-length streptokinase molecules.

\begin{tabular}{|ll|}
\hline Pf60 (forward) & 5'-CGAGGATCCAGTCCAAAATCAAAACC-3' \\
\hline Pf143 (forward) & 5'-ATAGGATCCCATGTGCGCGTTAGAC-3' \\
\hline Pr386 (reverse) & 5'-CCGCTGCAGTTACTAGGCTAAATGATAGCTAG-3' \\
\hline Pf1 (forward) & 5'-GAAGGATCCATTGCTGGACCTGAGTG-3' \\
\hline Pr414 (reverse) & 5'-ATCTGCAGTTATTTGTCGTTAGGGTTATCAGG-3' \\
\hline
\end{tabular}


inoculated in TY2X medium $\left(\mathrm{OD}_{600}=0.6\right)$ to express the recombinant protein for 3-5 hrs following induction by $0.1 \mathrm{mM}$ IPTG (Isopropyl-thiogalactoside).

Protein analysis and purification. Pellets of induced bacteria $(5 \mathrm{ml})$ were lysed in $50 \mu \mathrm{l}$ of lysis buffer $(100 \mathrm{mM}$ $\mathrm{NaH}_{2} \mathrm{Po}_{4}, 10 \mathrm{mM}$ Tris. $\mathrm{Cl}$ and $8 \mathrm{M}$ Urea), resuspended in $50 \mu \mathrm{l} \mathrm{Laemmli} \mathrm{buffer}(0.09 \mathrm{M}$ Tris- $\mathrm{HCl}, 20 \%(\mathrm{v} / \mathrm{v})$ Glycerol, 2\% (v/v) SDS, 0.02\% (v/v) bromophenol blue and $2 \%(\mathrm{v} / \mathrm{v}) \beta-\mathrm{ME})$ and heated at $80^{\circ} \mathrm{C}$ for $5 \mathrm{~min}$. The supernatants were electrophoresed in $12 \%$ SDSPAGE gel and separated proteins were either stained by coommasie Brilliant Blue or transferred to nitrocellulose membrane. After blocking the membrane in 3\% (w/v) bovine serum albumin (BSA) and application of proper dilution of mouse anti-His antibody (Qiagen, Germany) and washing steps, HRP-conjugated goat anti-mouse IgG (Sigma, USA) was utilized as tracking antibody. The bands related to the recombinant SK proteins were finally visualized by application of $\mathrm{DAB}$ (3.3' Diaminobenzidine) substrate.

Recombinant proteins harboring the N-terminally tagged 6xHis amino acids were purified in denaturing condition by the application of Nickel-TitriloTriacetic Acid (Ni-NTA) agarose columns (Qiagen, Germany), according to manufacturer protocol. Briefly, the pellets of $200 \mathrm{ml}$ of induced bacterial culture were lysed in $5 \mathrm{ml}$ of lysis buffer (100 mM NaH2Po4, $10 \mathrm{mM}$ Tris.Cl and $8 \mathrm{M}$ Urea) for 15 minutes. After centrifugation for 20 minutes at $10000 \mathrm{rpm}$, the upper lysate was mixed with $2 \mathrm{ml}$ of Ni-NTA resin for 30 minutes in a Falcon tube. The mixture was subsequently loaded into appropriate column (Qiagen, Germany), washed with $4 \mathrm{ml}$ of washing buffer (containing the same lysis buffer, $\mathrm{pH}$ 6.3) twice and finally the SK proteins were eluted by elution buffer (containing the same lysis buffer, $\mathrm{pH} 4.5$ ) in four fractions $(0.5 \mathrm{ml}$ each one). Purity and concentration of protein fractions were analyzed by SDS-PAGE and Bradford methods, respectively.

Bioassay of recombinant SK proteins. Streptokinase activity was determined by three methods:

Caseinolysis - In this semiquantitative standard method (Saksella, 1981) a plate containing 5\% (w/v) skim-milk, 1\% (w/v) agarose, $10 \mathrm{mM} \mathrm{NaCl}$ and $50 \mathrm{mM}$ Tris base was prepared and different dilutions of standard SK (B. Braun, Germany) and the same amounts of eluted proteins were applied in the $5-\mathrm{mm}$ wells previously prepared in the plate. All the standard and test wells were filled with $1 \mathrm{mg} \mathrm{ml}^{-1}$ concentration of plasminogen solution (Fluka, Sweden) to have the plasminogen in an excess molarity. Two other wells containing standard SK alone or plasminogen alone were also prepared as negative controls. After $24 \mathrm{~h}$ incubation at room temperature the caseinolysis diameter surrounding the wells, reflecting the functional activity of SK, was measured.
Chromogenic assay - This method that monitors the amount of formed plasmin by an endpoint assay of a synthetic substrate (British Pharmacopoeia, 1998) (Couto et al., 2004) was conducted with slight modifications. In brief, recombinant SK molecules $(0.4 \mathrm{nM})$ and Plg $(1 \mathrm{nM})$ were mixed together in 96-well plates and incubated at $37^{\circ} \mathrm{C}$ for $10 \mathrm{~min}$ to construct activator complexes. $0.75 \mathrm{mM}$ S2251 (H-D-valyl-L-leucyl-Llysine- $\rho$-nitroanalide) was added to the enzymatic complex as substrate and the mixture was incubated at $37^{\circ} \mathrm{C}$ for $20 \mathrm{~min}$. The reaction was stopped with $20 \%$ (v/v) acetic acid and absorbance was read in $405 \mathrm{~nm}$. Biological activity ( $\mathrm{IU} \mathrm{ml}^{-1}$ ) and specific activity ( $\mathrm{IU} \mathrm{mg}^{-1}$ and IU $\mathrm{nM}^{-1}$ ) of the samples were calculated according to $\mathrm{OD}_{405}$ of reference streptokinase with known biological activity (B. Braun, Germany).

Clot lysis assay - This method measures the SK activity in the presence of fibrin (British Pharmacopoeia, 1998). Briefly, $0.2 \mathrm{ml}$ of SK $(0.8 \mathrm{nM})$ (either reference or purified recombinant proteins) was mixed with $0.2 \mathrm{ml}$ of citric-phosphate buffer, $0.1 \mathrm{ml}$ of thrombin $\left(20 \mathrm{IU} \mathrm{ml}^{-1}\right)$ and $0.5 \mathrm{ml}$ of euglobulin $\left(10 \mathrm{mg} \mathrm{ml}^{-1}\right)$, which was purified according to the method used by Couto et al. (Couto et al., 2004), in a test tube. After the formation of clots, the required times for complete dissolution of clots was noted. Biological activity of the unknown purified samples was determined based on the plotted standard curve showing the clot lysis time against the $\mathrm{IU} \mathrm{ml} \mathrm{ml}^{-1}$ of different concentrations of reference SK. Biological activity $\left(\mathrm{IU} \mathrm{ml}^{-1}\right)$ and specific activity (IU mg $\mathrm{m}^{-1}$ and IU $\mathrm{nM}^{-1}$ ) of samples were calculated according to reference streptokinase plot with known biological activity (B. Braun, Germany).

Antibody preparation and Enzyme-Linked Immunosorbent Assay (ELISA). ELISA was used to evaluate antigenic discrepancy between full-length and truncated SK using polyclonal anti-SK antibodies generated in rabbits. Full length purified SK (SK1-414) emulsified in Complete Fraunds' Adjuvant (CFA, Sigma) was intramuscularly injected to six female New Zealand rabbits $(0.25 \mathrm{mg} /$ rabbit). Booster administration was performed with the same amount of SK mixed with Incomplete Fraunds' Adjuvant (IFA, Sigma) 4 wks later. The anti-sera were prepared at $4 \mathrm{wk}$ post-booster injection and used in an indirect ELISA to detect the truncated SK molecules. ELISA plate was coated with $0.4 \mathrm{nM}$ of the 3 proteins (SK143-386, SK60-386 and SK1-414). After blocking with 3\% (w/v) BSA, serial dilutions of anti-sera from individual injected rabbits $(1 / 800,1 / 1600,1 / 3200,1 / 6400,1 / 12800)$ were added to streptokinase coated wells. Following the washing steps, horse-radish peroxidase (HRP)-conjugated antirabbit antibody was applied to the wells. Finally and after further washes, addition of the chromogenic substrate; TMB (Tetramethyl-benzidine) led to the color 

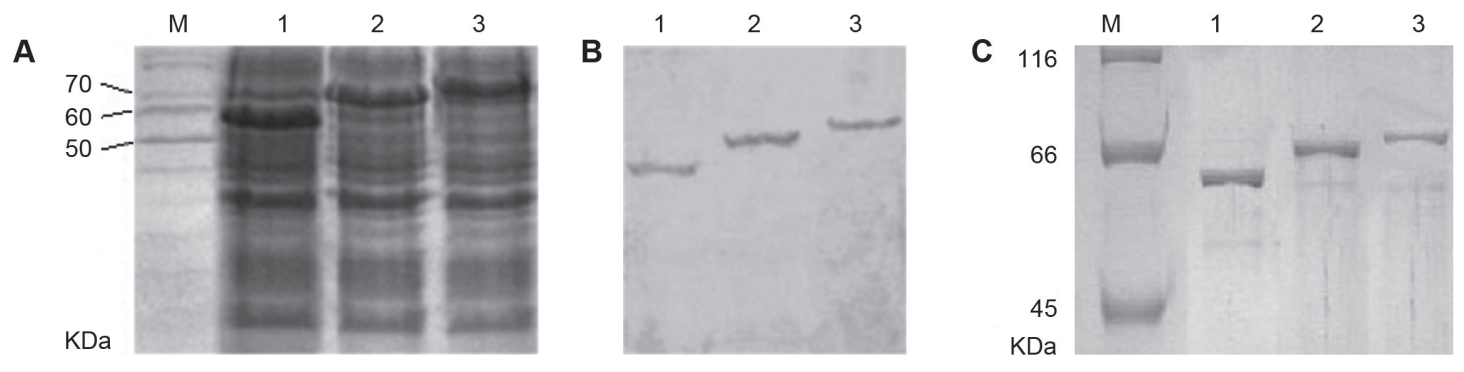

Fig. 2. Analysis of the expressed SK proteins.

(A) SDS-PAGE and (B) western blot analysis of crude lysis of E. coli BL21 cells expressing truncated and intact forms of streptokinase; lane M: molecular weight marker, lane 1-3: SK143-386, SK60-386 and SK1-414 respectively; (C) SDS-PAGE analysis of purified proteins; lane M: molecular weight marker, lane 1-3: SK143-386, SK60-386 and SK1-414 respectively.

appearance that was stopped by $10 \%(\mathrm{w} / \mathrm{v})$ acetic acid. While the background absorbance resulting from the pre-immune sera was subtracted from the tests, the means of $\mathrm{OD}_{450}$ for different groups were statistically compared using student $t$-tets.

\section{Results}

Modeling of truncated and full-length proteins. Expression of $\mathrm{N}$-terminally truncated proteins is shown to be usually less efficient (Nihalani et al., 1998; Reed et al., 1999). We hypothesized that expression of $\mathrm{N}$-terminally truncated SK proteins fused with $\mathrm{N}$-terminal tags (derived from vectors such as pET41a) that are efficiently expressed may be facilitated. Although Reed et al. (Reed et al., 1999) previously reported that maltose binding protein (MBP) fused to streptokinase did not affect its activity, however for us an existing concern with this strategy was the risk of unwanted changes in the configuration of these proteins. To gain insights into this possibility we took advantage of computerbased modeling using the MODELLER software.

To avoid complexity in modeling, the small 6His-tag (6 residues) and S-tag (15 residues) were ignored but GST tag, a protein with 220 amino acids was included in the modeling procedure. Several models were created by using 'multiple- template' scripts of MODELLER software and subsequently models with the lowest energy were selected for assessments (data not shown).

DOPE scores of models resulted from model evaluation scripts of MODELLER software were compared with DOPE scores of templates by depicting the data as plots (Fig. 3). There are gaps in the DOPE plots due to deletion of some residues in $1 \mathrm{bmlC}$ (template of SK), which is obtained from Protein Data Bank, however analogous points related to these gaps in the models show good level of energy (less than $-1 \mathrm{E}^{-2}$ ). Fortunately residues related to $\gamma$ domain, which is in close contact with catalytic site of plasminogen in the complex (Wang et al., 1998), in all 3 models of streptokinase had very near level of energy to their template (Fig. 3).
Assessment of selected models by Ramachandran plot showed that residues of outlier region for full-length SK (SK1-414), SK60-386 and SK143-386 were 4.9\%, 2.9\% and $3.5 \%$ respectively. These scores compared to the scores of their templates (6.1\% for $1 \mathrm{bmlC}$ and $1 \%$ for $1 \mathrm{~m} 9 \mathrm{~A}$ ) seemed very reasonable.

RMSD of selected models for the 3 proteins after superimposing to $1 \mathrm{bmlC}$ as template for streptokinase and from $1 \mathrm{~m} 9 \mathrm{aA}$ as template GST-tag, were calculated separately. These results indicated that SK143-386 model has the least RMSD in comparison to the other models which means it was the best model (RMSD less than $2 \AA$ is considered as excellent, between 2 and $6 \AA$ as reasonable and more than $6 \AA$ as improper). Altogether the results of all three kinds of evaluations showed that the fusion tags may not have considerable impact on the conformation of our proteins.

Cloning, expression and purification of recombinant full length and truncated streptokinase proteins. Two truncated SK genes encoding 143-386 and 60-386 amino acid residues (skc143-386 and skc60-386, respectively) in addition to the full-length SK gene (skc1-414) were PCR-amplified from the previously isolated $s k c 2$ template (Estrada et al., 1992). The amplicons were cloned into the BamH1/Pst 1 sites of pET41a plasmid, in the same open reading frame (ORF) of vector-born $\mathrm{N}$-terminal fusion-tag (Fig. 1). Transformation and subsequent expression by IPTG induction of plasmids in E. coli BL21 (DE3) resulted in the appearance of protein bands with expected molecular weights for vector derived tags fused to the proteins $(57,66$ and $78 \mathrm{kDa}$ for SK143-386, SK60-386 and SK1-414, respectively) in SDS-PAGE (Fig. 2A) and Western-blot analyses (Fig. 2B).

Induction of protein expressions in large scale cultures $(200 \mathrm{ml})$ and purification of His-tagged SK proteins using Ni-NTA affinity chromatography finally provided us with approximately $150 \mathrm{mg}$ of full length and truncated proteins with a purity of more than 90 percent for each protein that was shown by SDSPAGE (Fig. 2C). These proteins were further evaluated for bioactivity and antigenicity. 

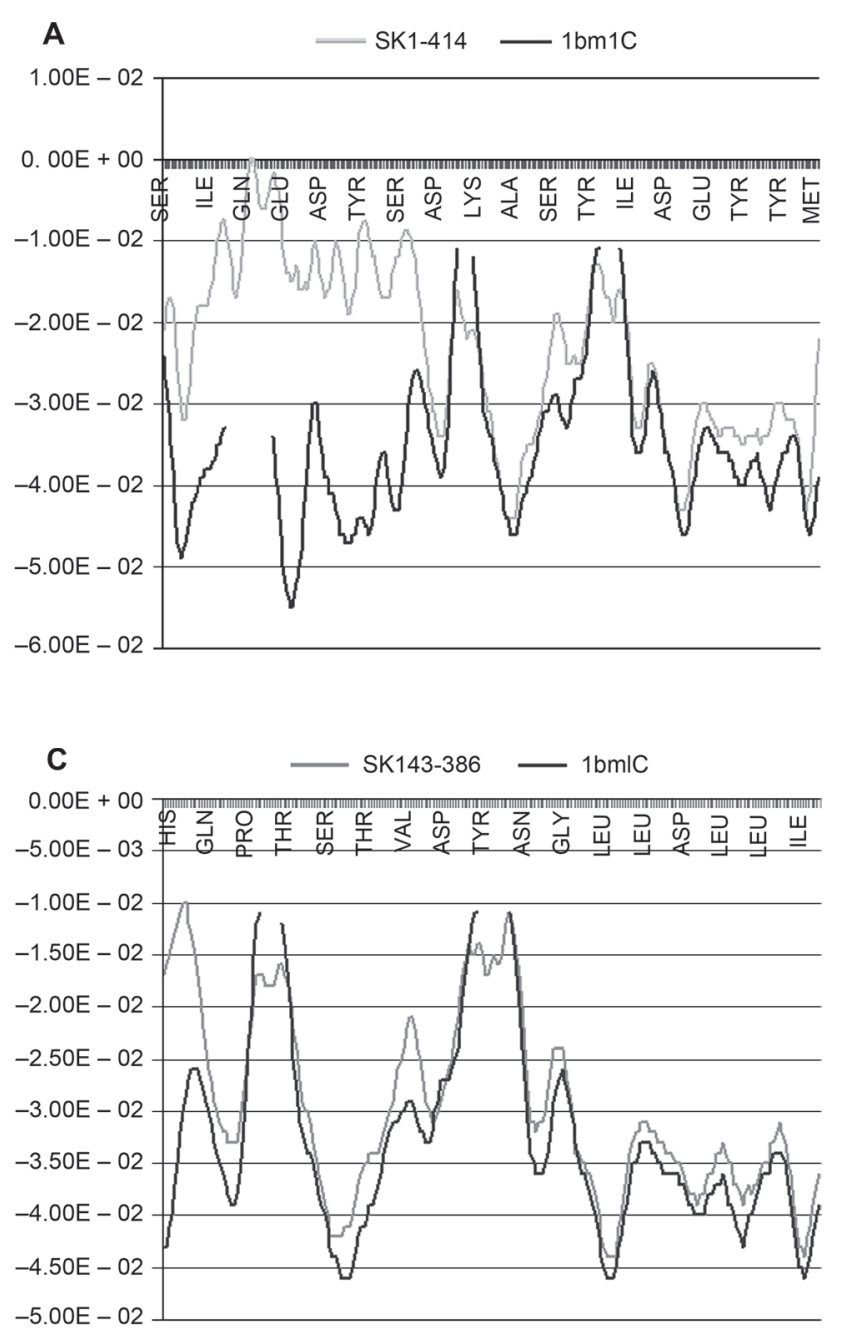

Comparison of biological activity for truncated and full length purified SK proteins. The caseinolysis method (Saksella, 1981) was used for preliminary activity assessment of the recombinant SK proteins. Two other methods used in this study were chromogenic plasminogen activation assay and a fibrin clot lysis assay which are recommended assays for biological activity assessment of streptokinase in British Pharmacopoeia (BP, 1998). Caseinolysis zones, surrounding the wells of samples together with different amounts of standard streptokinase, indicated that full length and truncated forms of SK proteins were biologically active (data not shown). Following this primary screening, specific activities of proteins were measured in terms of International Unit per milligram and per nanomole. The quantitative chromogenic assay indicated that, compared to SK1-414, specific activities of SK60-386 and SK143-386 had respectively reduced by 81 and 88 percent in terms of IU mg ${ }^{-1} 83$ and 91 percent in terms of IU nM-1 (Table II). Similarly, the clot lysis assay (lysis of one milliliter of clot by one microgram or nanomole streptokinase) showed a reduction of 68 and $80 \%$ of $\mathrm{IU} \mu \mathrm{g}^{-1}$ and 72 and $85 \%$ of IU $\mathrm{nM}^{-1}$, respectively, compared to SK1-414 (Table III). This method that mea-

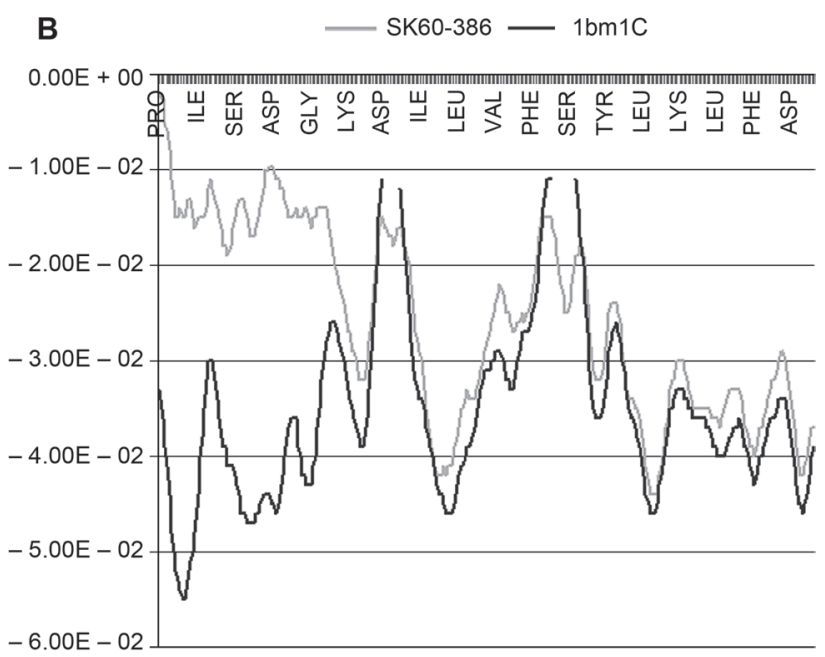

Fig. 3. DOPE score profiles of streptokinase models.

A: SK1-414, B: SK60-386, C: SK143-386; all three models totally show good (almost residues less than $-1.00 \mathrm{E}^{-2}$ ) energy level when compared to their template, especially in $\mathrm{C}$ terminus which has close contact with catalytic site on plasminogen. The gaps in some parts of DOPE plot of template are related to deletion of some residues of template downloaded from Protein Data Bank (1bmlC).

sures the activity of SK in the presence of fibrin may be considered as a criterion for the fibrin dependency of SK molecule in physiologic condition (Reed et al., 1999). Of note, all SK proteins (both full length and truncated SKs) were able to completely lyse the clots during 20 minutes which was acceptable and allowed time for a thrombolytic drug with potential therapeutic

Table II

Biologic and specific activity of truncated and full-length streptokinase molecules measured by chromogenic method.

\begin{tabular}{|l|c|c|c|}
\hline Biological activity $\left(\mathrm{IU} \mathrm{ml}^{-1}\right)$ & $\begin{array}{c}\text { SK1-414 } \\
3758\end{array}$ & $\begin{array}{c}\text { SK60-386 } \\
634\end{array}$ & $\begin{array}{c}\text { SK143-386 } \\
346\end{array}$ \\
\hline Specific activity $\left(\mathrm{IU} \mathrm{mg}^{-1}\right)$ & 11743 & 2186 & 1443 \\
\hline Specific activity $\left(\mathrm{IU} \mathrm{nMol}^{-1}\right)$ & 952 & 158 & 88 \\
\hline
\end{tabular}

Table III

Biologic and specific activity of truncated and full-length streptokinase molecules measured by clot lysis method.

\begin{tabular}{|l|c|c|c|}
\hline & SK1-414 & SK60-386 & SK143-386 \\
\hline Biological activity $\left(\mathrm{IU} \mathrm{ml}^{-1}\right)$ & 3211 & 1464 & 1011 \\
\hline Specific activity $\left(\mathrm{IU} \mathrm{mg}^{-1}\right)$ & 10703 & 3405 & 2106 \\
\hline Specific activity $\left(\mathrm{IU} \mathrm{nMol}^{-1}\right)$ & 868 & 244 & 128 \\
\hline
\end{tabular}



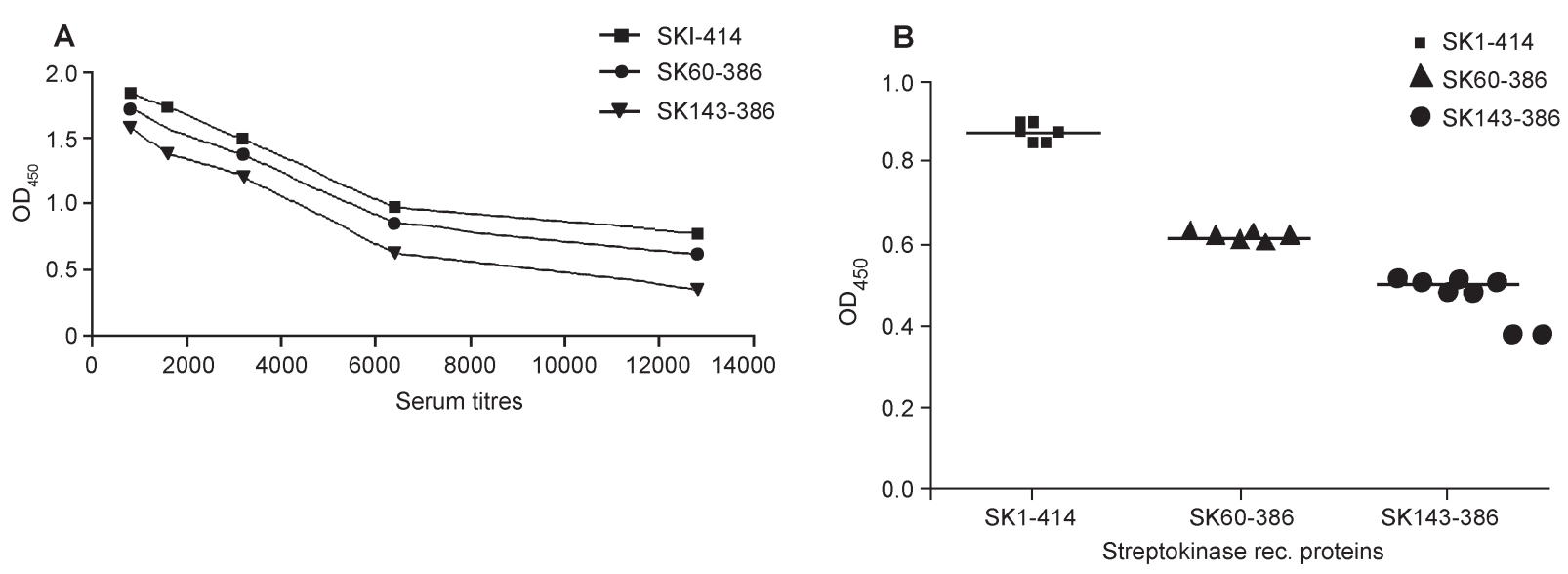

Fig. 4. Antigenicity analysis of truncated forms of streptokinase in comparison to full length SK.

(A) Reactivity of five serial dilutions of anti-SK polyclonal antisera on SK143-386, SK60-386 and SK1-414 by indirect ELISA. (B) Comparison of ELISA signals obtained with 1/12800 dilution of antisera in pairs of SK1-414 \& SK143-386, SK1-414 \& SK60-386 and SK60-386 \& SK143-386. Deletion of residues 1-59, 1-142 and 387-414 in streptokinase resulted in significantly less antigenic proteins ( $\mathrm{p}<0.0001$ for all comparisons).

efficacy in the British pharmacopoeia. Comparison of the results obtained from clot lysis and chromogenic assays (in terms of $\mathrm{IU} \mathrm{nM}^{-1}$ ) also indicated that while the specific activity of full length SK in the presence of fibrin was reduced approximately by $9 \%$, but both truncated molecules showed an increased activity of 35\% and 31\% for SK60-386 and SK143-386 respectively.

Although there was significant difference in the specific activity between truncated and full-length streptokinase it was observed that by equal amount (0.8 nM), SK60-386 and SK143-386 completely lysed the clot approximately in 12 and 16 minutes, respectively, whereas SK1-414 solubilized the clot in about 3 minutes; i.e. the truncated molecules had full activity in longer times but still in the range of allowed time for a thrombolytic drug with potential therapeutic efficacy in the British pharmacopoeia (20 minutes).

Antigenicity analysis of full length and truncated SK molecules. Immunological characteristics of streptokinase can be evaluated by developing anti-SK antisera in different animals, including rabbits (Houba and Hana, 1966) and monkeys (Torrèns et al., 1999). In this study for comparison of antigenicity of the proteins, rabbit polyclonal antisera raised against purified SK1-414, were used to analyze antigenicity of truncated streptokinase proteins. Reactivity of five serial dilutions $(1 / 800,1 / 1600,1 / 3200,1 / 6400$ and $1 / 12800)$ of the antisera with SK143-386, SK60-386 and SK1-414 which was measured by indirect ELISA pointed to lower signals $\left(\mathrm{OD}_{450}\right)$ in the case of truncated molecules (Fig. 4A). Dilution of $1 / 12800$ was selected to statistically compare the observed differences of antigenicity between the 3 proteins (Fig. 4B). Interestingly, this analysis showed a significant decrease of reactivity for both truncated molecules in comparison with full length SK. SK143-386 showed to be $45 \%$ less reactive to antiSK than SK1-414 $(\mathrm{P}<0.0001)$ and SK60-386 had 28\% lower reactivity in comparison to SK1-414 $(\mathrm{P}<0.0001)$. Also reactivity of SK60-386 to anti-SK was 23\% higher than SK143-386 ( $<<0.0001)$. These data, in accordance with previous study (Torrèns et al., 1999) on evaluation of $\mathrm{C}$ terminally mutated streptokinase, indicated that removal of the selected fragments ( 59 or 142 residues of $\mathrm{N}$ terminal and 28 residues of $\mathrm{C}$ terminal) from full length SK leads to the production of considerably less immunogenic SK derivatives.

\section{Discussion}

Streptokinase is one of the most important drugs for thrombolytic therapy; however, it has shortcomings such as immunogenicity and fibrin-non specificity (Banerjee et al., 2004; Baruah et al., 2004; Reed et al., 1999; Sazonova et al., 2004). In the present study, based on previous reports on antigenic mapping and functional characteristics of SK regions, two truncated SK-molecules lacking the $59 \mathrm{~N}$-terminal or the $142 \mathrm{~N}$-terminal amino acids plus $28 \mathrm{C}$-terminal residues (SK60-386 and SK143-386 respectively) were considered for construction and analysis of antigenicity/ activity. To the best of our knowledge there was no prior report on evaluation of activity of SK143-386 and SK60386 as potential thrombolytic drugs for their therapeutic efficacy by a fibrin based and pharmacopoeia approved method. Further, no prior study addressed for comparison of the antigenicity of these truncated SK proteins with full-length SK.

Bioassay of truncated SK proteins by chromogenic method indicated a dramatic decline of activity compared to the full length SK (up to 84 and 91 percents for SK60-386 and SK143-386, respectively (Table II). According previous reports, on structure/function analysis of streptokinase, this lower activity of truncated SK 
proteins in chromogenic assay was expectable (Reed et al., 1999; Rodriguez et al., 1995). Analysis by fibrin clot lysis assay also evidenced for significant activity reduction for truncated SK proteins compared to fulllength SK (Table III), but comparison of activities by two methods (Chromogenic assay versus fibrin clot assay) indicated $35 \%$ and $31 \%$ increase of activity for SK60-386 and SK143-386 respectively and reduction of $9 \%$ for full-length SK by clot lysis assay compared to chromogenic assay (Tables II, III). These observations are somehow in accordance with prior studies which demonstrated SK lacking $\mathrm{N}$ terminal 59 amino acids restores the activity in the presence of fibrin, however in the present study the truncated SK proteins did not restored full activity. Accordingly, while it was already shown that during a long analyzing time (6 hours) SK60-414 is more active than full-length SK in all ranges of the tested concentrations (0-50 nM), (Reed et al., 1999), other studies showed that SK60-414 is superior than full-length SK only in high concentrations (Mundada et al., 2003). The results of our clot lysis test are consistent with the later report in that our truncated proteins had less activity than full-length SK using a lower concentration $(0.8 \mathrm{nM})$.

The lag time in fibrinolysis, which was the major reason for lower activity in our clot lysis assay, may be explained by the fact that both truncated molecules (lacking 59 first residues) were only able to form efficient activator complexes with plasmin and not with plasminogen (Mundada et al., 2003; Sazonova et al., 2004) which were generated in trace amount in the beginning of the interaction. By more plasmin formation, further plasminogen substrates would be subsequently activated in a time manner. Delay in fibrin degradation can be, in fact, a positive feature, since it gives the opportunity to SK to become active only when it reaches to the loci occluded by fibrin clots. This feature can minimize activity of the SK protein in the regions lacking fibrin clots and may potentially reduce the risk of hemorrhage.

The other side of our rationale for the truncation of streptokinase was to reduce its antigenicity. Antigenicity analysis by ELISA which was carried out in our study was based on methods previously developed for evaluation of the immunological characteristics of streptokinase using anti-SK antisera developed in different animals, including rabbits (Houba and Hana, 1966 and Torrèns et al., 1999). These analyses showed a less reactivity of the SK-specific rabbit polyclonal antisera against the truncated SK molecules (Fig. 5), so that a significant decrease in the obtained signals was observed for truncated molecules in comparison with the full length protein, which was still significantly lower for SK143-386 compared to the SK60-386. These findings were somehow expected and compatible with other studies (Parhami-Serena et al., 2003; Reed et al., 1993; Torrèns etal., 1999) which mapped and analyzed antigenic epitopes of streptokinase. Accordingly, lower reaction of anti-SK1-414 antibodies with truncated molecules confirmed the accumulation of antigenic determinants in the excised regions. Moreover, in a confirmatory experiment we prepared anti-truncated SK antibodies by immunizing the rabbits with SK60-386 and SK143-386 molecules and found that in comparison with anti-full length SK antibodies, antisera raised against the shortened molecules generally had a lower reactivity with their corresponding proteins (data not shown). Overall, these findings verified the less antigenicity/immunogenicity of the truncated SK proteins introduced in this study.

Altogether, based on the available structure/function and antigenic mapping reports of SK molecule, to our knowledge the present study for the first time attempted to engineer truncated recombinant SK molecules with a simultaneous more fibrin specificity and less antigenicity. According to the obtained results, truncation of SK in both $\mathrm{N}$ - and C-terminal ends was successful to create fibrin targeted SKs with comparable activities and considerably lower antigenic properties. In a preliminary analysis, both of these proteins (SK143-386 and SK60-386) passed the pharmacopoeia standard for streptokinase activity assessment by a clot lysis assay for evaluation of a thrombolytic drug with potential therapeutic efficacy and hence may be considered for further pharmacological assessments.

\section{Acknowledgments}

This study was financially supported by the Education Office of Pasteur Institute of Iran. The authors would like to thank Mr. Hendi for his technical assistance in biological activity assays. This paper is a partial fulfillment of a Ph.D. thesis by R.A.

\section{Literature}

Banerjee A., C. Chisti and U.C. Banerjee. 2004. Streptokinase - a clinically useful thrombolytic agent. Biotechnol. Adv. 22: 287-307.

Baruah D.B., R.N. Dash, M.R. Chaundhari and S.S. Kadam. 2004. Plasminogen Activators: A comparison. Vascul. Pharmacol. 44: 1-9. Coffey J.A., K.R. Jennings and H. Dalton. 2001. New antigenic regions of streptokinase are identified by affinity directed mass spectrometry. Eur. J. Biochem. 268: 5215-5221.

Conjero-Lara F., J. Parrado, A.I. Azuaga, C.M. Dobson and P.C. Paula. 1998. Analysis of the interactions between streptokinase domains and human plasminogen. Protein Sci. 7: 2190-2199.

Couto L., J.L. Donato and G. Nucci. 2004. Analysis of five streptokinase formulations using the euglobulin lysis test and the plasminogen activation assay. Brazil J. Med. Biol. Res. 37: 1889-1894.

Estrada M., L. Hernández, A Pérez, P. Rodríguez, R. Serrano, R. Rubiera, A.Pedraza, G. Padrón, W. Antuch, J. de la Fuente and L. Herrera. 1992. High level expression of streptokinase in Escherichia coli. Biotechnol. Adv. 10: 1138-1142. 
Houba V. and I. Hana. 1966. The difference in immunological characteristics of two streptokinases. Immunol. 11: 387-397.

Lovell S.C., I.W. Davis, W.B. Arendall, P.I.W. de Bakker, J.M. Word, M.G. Prisant, J.S. Richardson and D.C. Richardson. 2002. Structure validation by Calpha geometry: phi,psi and Cbeta deviation. Proteins: Struct. Funct. and Genet. 50: 437-450.

Mundada L.V., M. Prorok, M.E. DeFord, M. Figuera, F.J. Castellino and W.P. Fay. 2003. Structure-function analysis of the streptokinase amino terminus (residues 1-59). J. Biol. Chem. 278: 24421-24427.

Nihalani D., R. Kumar, K. Rajagopal and G. Shan. 1998. Role of the amino-terminal region of streptokinase in the generation of a fully functional plasminogen activator complex probed with synthetic peptides. Protein Sci. 7: 637-648.

Parhami-Seren B., K. Keel and G.L. Reed. 1997. Sequences of antigenic epitopes of streptokinase identified via random peptide libraries displayed on phage. J. Mol. Biol. 271: 333-341.

Parhami-Serena B., M. Lynch, H.D. Whites and G.L. Reed. 1995 Mapping the antigenic regions of streptokinase in humans before and after streptokinase therapy. Mol. Immunol. 32: 111-724.

Parhami-Serena B., M. Seaveya, J. Krudysza and P. Tsantili. 2003. Structural correlates of a functional streptokinase antigenic epitope: serine 138 is an essential residue for antibody binding. J. Immunol. Methods. 272: 93-105.

Reed G., P. Kussie and B. Parhami-Seren. 1993. A functional analysis of the antigenicity of streptokinase using monoclonal antibody mapping and recombinant streptokinase fragments. The J. Immunol. 150: 4407-4415.
Reed G., B. Parhami-Seren, S. Wang and L. Hedstrom. 1999. A catalytic switch and the conversion of streptokinase to a fibrin targeted plasminogen activator. PANAS 96: 8879-8883.

Rodriguez P., P. Fuentes, M. Barro, J.G. Alvarez, M. Mwozz, D.C. Collen and H.R. Lunen. 1995. Structural domains of streptokinase involved in the interaction with plasminogen. Eur. J. Biochem. 229: 83-90.

Saksella O. 1981. Radial caseinolylis in agarose: a simple method for detection of plasminogen activator in the presence of inhibitory substances and serum. Anal. Biochem. 111: 276-282.

Sazoana I.Y., R.A. Mcanmee, A.K. Houng, S.M. King, L. Hedstorm and G.L. Reed. 2009. Reprogrammed streptokinases develop fibrin-targeting and dissolve blood clots with more potency than tissue plasminogen activator. J. Thromb. and Haemostasis 7: 1321-1328. Sazonova I.Y., B.R. Robinson, I.P. Gladysheva, F.J. Castellino and G.L. Reed. 2004. Alpha domain deletion converts streptokinase into a fibrin-dependent plasminogen activator through mechanisms akin to staphylokinase and tissue plasminogen activator. J. Biol. Chem. 279: 24994-5001.

Shi G., B. Chang, S. Chen, D. Wu and H. Wu. 1994. Function of streptokinase fragments in plasminogen activation. Biochem. J. 304: 235-241.

Torrèns I., A.G. Ojalvo, A. Seralena, O. Hayes and J. Fuente. 1999. A mutant streptokinase lacking the C-terminal 42 amino acids is less immunogenic. Immunol. Lett. 70: 213-218.

Wang X., X. L i n, J.A. Lay, J. Tang and X.C. Zhang. 1998. Crystal structure of the catalytic domain of human plasmin complexed with streptokinase. Science 281: 1662-1665. 